\title{
PROBLEM DECOMPOSITION AND INFORMATION MINIMIZATION FOR THE GLOBAL, CONCURRENT, ON-LINE VALIDATION OF NEUTRON NOISE SIGNALS AND NEUTRON DETECTOR OPERATION
}

\author{
Tatiana Tambouratzis ${ }^{1}$ \\ Department of Industrial Management \& Technology, University of Piraeus, 107 \\ Deligiorgi St., Piraeus 185 34, Greece
}

\begin{abstract}
This piece of research introduces a purely data-driven, directly reconfigurable, divide-and-conquer on-line monitoring (OLM) methodology for automatically selecting the minimum number of neutron detectors $(N D s)$ - and corresponding neutron noise signals (NSs) - which are currently necessary, as well as sufficient, for inspecting the entire nuclear reactor (NR) in-core area. The proposed implementation builds upon the 3-tuple configuration, according to which three sufficiently pairwise-correlated NSs are capable of on-line (I) verifying each NS of the 3-tuple and (II) endorsing correct functioning of each corresponding $N D$, implemented herein via straightforward pairwise comparisons of fixed-length sliding time-windows (STWs) between the three NSs of the 3-tuple. A pressurized water NR (PWR) model-developed for H2020 CORTEX - is used for deriving the optimal ND/NS configuration, where (i) the evident partitioning of the $36 \mathrm{NDs} / \mathrm{NSs}$ into six clusters of six NDs/NSs each, and (ii) the high cross-correlations (CCs) within every 3-tuple of NSs, endorse the use of a constant pair comprising the two most highly CC-ed NSs per cluster as the first two members of the 3-tuple, with the third member being each remaining NS of the cluster, in turn, thereby computationally streamlining OLM without compromising the identification of either deviating NSs or malfunctioning NDs. Tests on the in-core dataset of the PWR model demonstrate the potential of the proposed methodology in terms of suitability for, efficiency at, as well as robustness in ND/NS selection, further establishing the "directly reconfigurable" property of the proposed approach at every point in time while using one-third only of the original NDs/NSs.
\end{abstract}

\section{KEYWORDS}

On-Line Monitoring (OLM), Pressurized Water (nuclear) Reactor (PWR), in-core Neutron Noise Detector/Signal (in-ND/NS) Validation, 3-Tuple Configuration, Computational (time/space) Efficiency

\section{INTRODUCTION}

The aim of this piece of research is to identify the minimal set of in-core neutron detectors (inNDs) - and corresponding in-core neutron noise (NN) signals (in-NSs ${ }^{2}$ ) - which, when configured into the minimum necessary number of in-ND/NS 3-tuples [1], constitutes a necessary

\footnotetext{
Dedication: This piece of research is dedicated to the COVID-19 victims worldwide.

${ }^{1}$ Acknowledgements: The research leading to these results (i) has received funding from the H2020 CORTEX Euratom Research and Training programme 2014-2018 under grant agreement No 754316, (ii) is supported by the Research Office of the University of Piraeus; the author wishes to thank both institutions for their support and for providing a strong initiative for research.

${ }^{2}$ as captured by the in-NDs, with each in-ND recording/monitoring one in-NS and each in-NS providing the measurements captured by the corresponding in-ND
} 
as well as sufficient set of in-NDs/NSs for collectively, on-line monitoring (OLM) the nuclear reactor (NR) core, in terms of

(I) valid operation of each in-ND and

(II) "correctness" 3 of each corresponding in-NS.

The proposed methodology is based on the combination of two straightforward signal processing (SP) techniques, namely:

(i) the application of cross-correlation (CC) [2] to the shortest possible (of length 64) sliding by 1 - time-window (STW) ${ }^{4}$ of appropriately selected (so as to be sufficiently similar) inNS pairs, for acquiring salient on-line information concerning the pairwise agreement (either synchronized, or with a NR operation- and/or time-dependent delay) between the constituent in-NSs,

(ii) the methodological selection of the smallest possible number of in-NSs (acquired by the corresponding in-NDs) which, when configured into the minimal set of sufficiently correlated 3-tuples of in-NSs, is capable of on-line determining (I) and (II) over the entire in-core area as well as for any time-window of practical interest.

A pilot investigation of the proposed methodology has been tested successfully [3] on the ex-core NS dataset of a pressurized water NR (PWR) [4] model [5] created for H2020 CORTEX [6]. The implementation employed the incremental/recursive application of the harmony theory artificial neural network architecture (HTN) [7] for the concurrent optimization of (I) and (II), as well as for the minimization of OLM time/space complexity, both of which have been accomplished - in the specific case - via the use of only (a) four (out of the total of eight, i.e. 50\% reduction in employed) NSs/NDs, configured into (b) two (out of the 56 combinatorially possible, i.e. $96.4 \%$ reduction in employed) 3-tuples, for the effective and successful OLM of the entire NR ex-core area.

This piece of research builds upon the general concept of [3], adapted to - and demonstrated on the corresponding in-core NN dataset of the same PWR model, thus further

- transferring the successful validation of the smaller set of (eight) ex-core NDs and NSs to the significantly augmented set of 36 in-core (in-)NDs and (in-)NSs of the same PWR model,

- confirming the scalability of the 3-tuple configuration to a model which demonstrates significantly more varied characteristics - in terms of both ND locations and range of CC values - over the entire set of in-NS, as well as more similar CC values between the strongest candidates for inclusion in the same 3-tuple of in-NSs, i.e. in all,

endorsing the validity of each in-NS, and the correct operation of each corresponding in-ND in every cluster through the use of 3-tuple(s) which contain a constant common pair of the two most highly CC-ed in-NSs of the cluster, with the third member of every created 3-tuple being each remaining NS of the cluster, in turn, thereby computationally streamlining OLM without compromising the identification of either deviating NSs or malfunctioning in-NDs ${ }^{5}$, thus - in all - configuring an in-ND/in-NS set-up that is not only sufficient under normal/steady operating conditions, but also directly adaptable/reconfigurable under rapidly changing

\footnotetext{
${ }^{3}$ in terms of concerted agreement of the in-NSs of the 3-tuple with their expected/reference values

${ }^{4}$ for the specific dataset and scenario

${ }^{5}$ with the validation of correct operation/collected values of the two constant NDs/NSs, respectively, being implemented automatically at each time-step via the respective CCs
} 


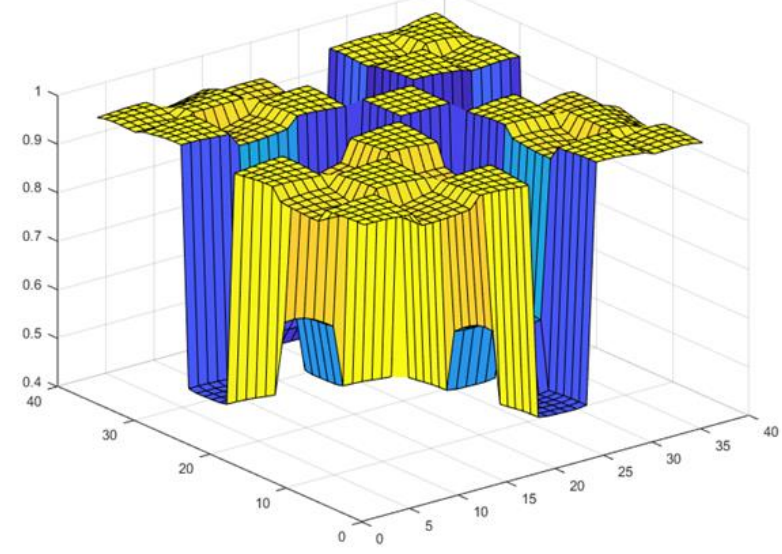

(a)

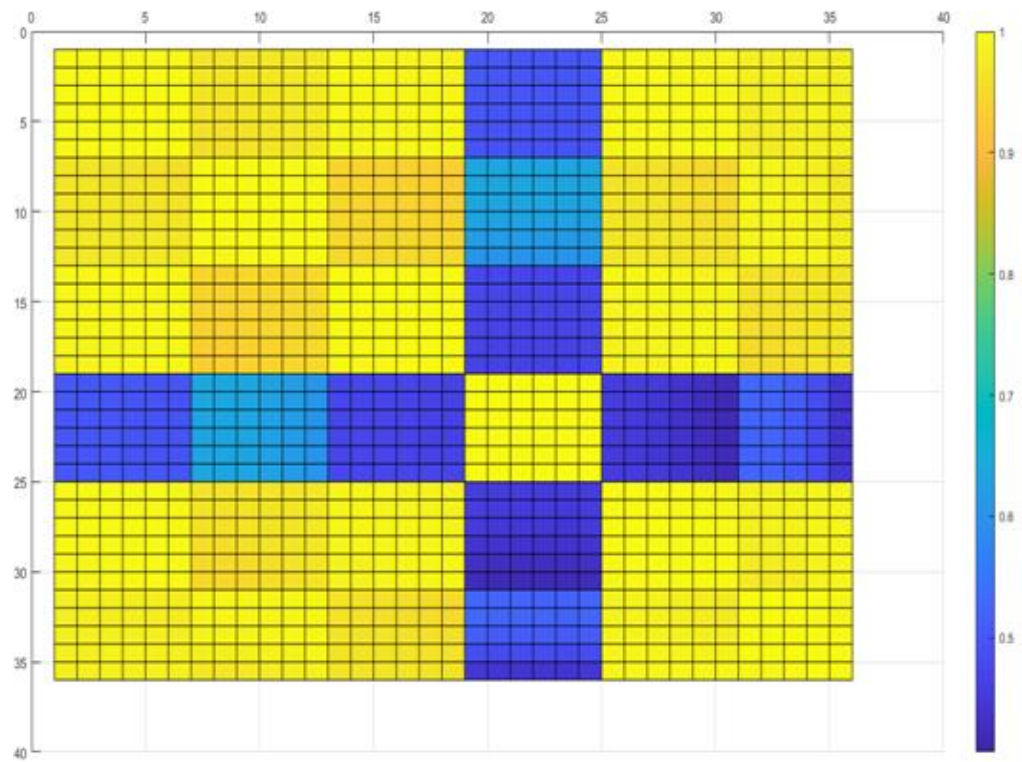

(b)

Figure 1. Three- (a) and two- (b) dimensional plots of the CCs between the 36 in-NSs, as captured by the corresponding in-NDs over the entire signal length of the in-NSs, revealing (A) high CCs within each of the groups of six proximal in-NSs/ in-NDs (thus constituting well-defined areas of similar information content), and (B) independent in-ND capture of in-NSs between groups located in different in-core NR areas.

NR operating conditions (e.g. transient operation, abnormal ND function/NS collection).

The remainder of this contribution is organized as follows: Section 2 introduces the in-ND/NS dataset used for demonstrating the proposed concept and OLM system, exposing salient statistical characteristics of the in-NSs per se, as well as of the relationships between in-NSs belonging to the same and to different sub-areas of the NR. Section 3 presents an improved version of the original 3-tuple methodology, which is based on a constant pair of NSs/NDs for each to-becreated 3-tuple of NDs/NSs, where (A) the selection of the NS pair is based on the cumulative degree to which each NS satisfies the criteria of highest CC values for (a) the minimum, (b) the maximum and (c) the mean CC value with the other NSs, as well as the lowest value for (d) the 
std of the CC value with the other NSs; such a choice encourages the selection of NSs/NDs which have both high and similar CCs with the remaining in-ND/NS, respectively, thus also showing the highest differences in the responses of the 3-tuple between deviating in-NSs and failing in-NDs. Tests on the in-core dataset of the PWR model demonstrate the potential of the proposed methodology in terms of suitability, efficiency and robustness in ND/NS selection, while further establishing the "directly reconfigurable" property of the proposed approach at every point in time using only one-third of the original NDs/NS. Section 4 critically summarizes the main findings and concludes with future extensions of the presented OLM implementation.

\section{Problem Presentation \& Data Analysis}

\subsection{In-Core Area Partitioning - Central and Peripheral Areas}

The in-core PWR dataset ([5], Scenario 1), which has been used here for validating the proposed methodology, contains 36 in-NSs, each of length 3501, collected simultaneously by as many in$\mathrm{NDs}^{6}$ which have been strategically installed in the in-core area. As illustrated in fig. 1(a-b), the pairwise CCs between the 36 in-NSs reveal the following interesting characteristics:

(A) Monitored by the in-NDs, the in-core area (fig. 1) is partitioned into two practically uncorrelated (CC $\in \quad$ [0.4056 0.6417]) sub-areas, with one partition (the central NR area) comprising NDs/NSs 19 through to 24 and the other partition/sub-area (the peripheral NR area) including NDs/NSs 1 through to 18 and 25 through to 36 .

(B) The central NR area (fig. 2) comprises six highly CC-ed in-NSs (CCs $€$ [0.9991 1.000]), with NS pairs $\{19,20\},\{20,21\},\{19,21\}$ and $\{23,24\}$ returning maximum CCs (of 1); the minimum CC value of 0.9991 , which is observed for NS pair $\{19,24\}$, still constitutes a significant - and more than sufficient - CC value for the application of the 3-tuple configuration (Fig. 2(a)), with the largest $(<1) \mathrm{CC}$ values for this area encountered next to the main diagonal of the CC matrix (Fig. 2(b)).

(C) The peripheral NR area contains five $6 \times 6$, evidently separate from each other, sub-areas of highly CC-ed NSs, all of which are located along the main diagonal; the partitioning "pattern" is guided by the highly CC-ed pairs of NSs within each sub-area (maximum CCs of 0.9999 for all sub-areas and minimum CCs of 0.9946, 0.9944, 0.9939, 0.9926 and 0.9864 for each $6 \times 6$ sub-area, respectively). Furthermore, the CCs within each of the entire 1-18 and 25-36 areas remain significantly high, ranging in [0.9314 0.9999] and [0.9727 0.9999], respectively ${ }^{7}$. Conversely, the CCs between the entire NR 1-18 \& 19-24 areas range in [0.4650-0.6417], those between the entire NR 1-18 \& 25-36 areas in [0.40650.5277], and those between the entire NR 19-24 \& 25-36 areas in [0.4056-0.5277], thus indicating that combining information from any given pair of different areas does not provide pertinent information for the OLM-related purposes of ND/NS selection.

\subsection{Proposed Problem Decomposition}

For the scenario used here, both the CCs between in-NSs and (consequently) the pairwise ratios between in-NS synchronized pairs of NSs remain practically invariable (constant) over the entire time-window of in-NS capture. Combined with the well-partitioned structure of the NR, the two observations call for a straightforward and computationally light custom-made divide-and-

\footnotetext{
${ }^{6}$ for more details on the exact in-ND locations as well of the NS perturbation characteristics, the interested reader is referred to [5]

${ }^{7}$ it should be mentioned that, although - due to their high CC values -, the 25th-36th ND/NS could be treated as a single area for further reducing OLM complexity, the two partitions have been retained here for generality of treatment of the NR areas via the $6 \times 6$ configuration
} 
International Journal of Artificial Intelligence and Applications (IJAIA), Vol.11, No.5/6, November 2020

conquer (problem decomposition) methodology, which eliminates the need for detailed comparisons, either between alternative candidate states (solutions), or across extensive parts of the NR area per se. For the present OLM implementation, the six 6x6 sub-areas of highly CC-ed NSs which lie along the main diagonal remain sufficiently dissimilar from each other, whereby they have been processed concurrently, yet independently of each other, for performing minimalresource OLM that remains capable of ranging over the entire in-core NR area.

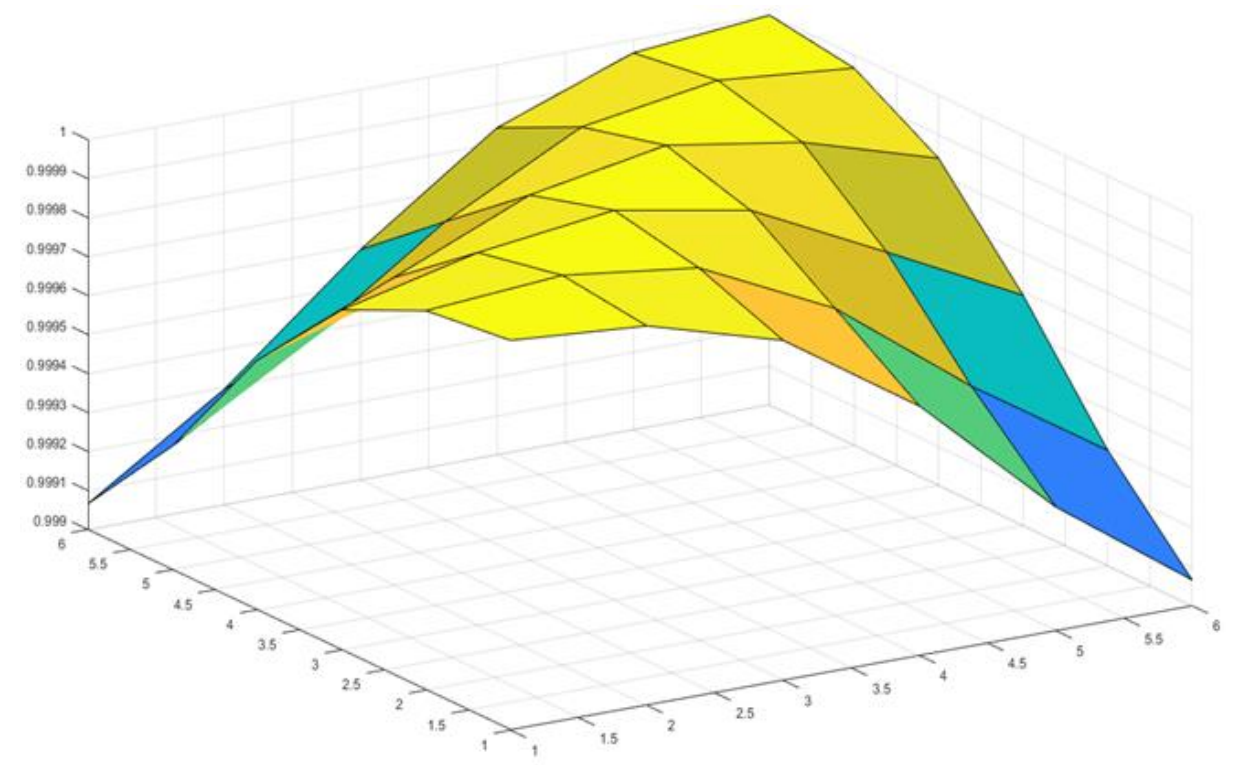

(a)

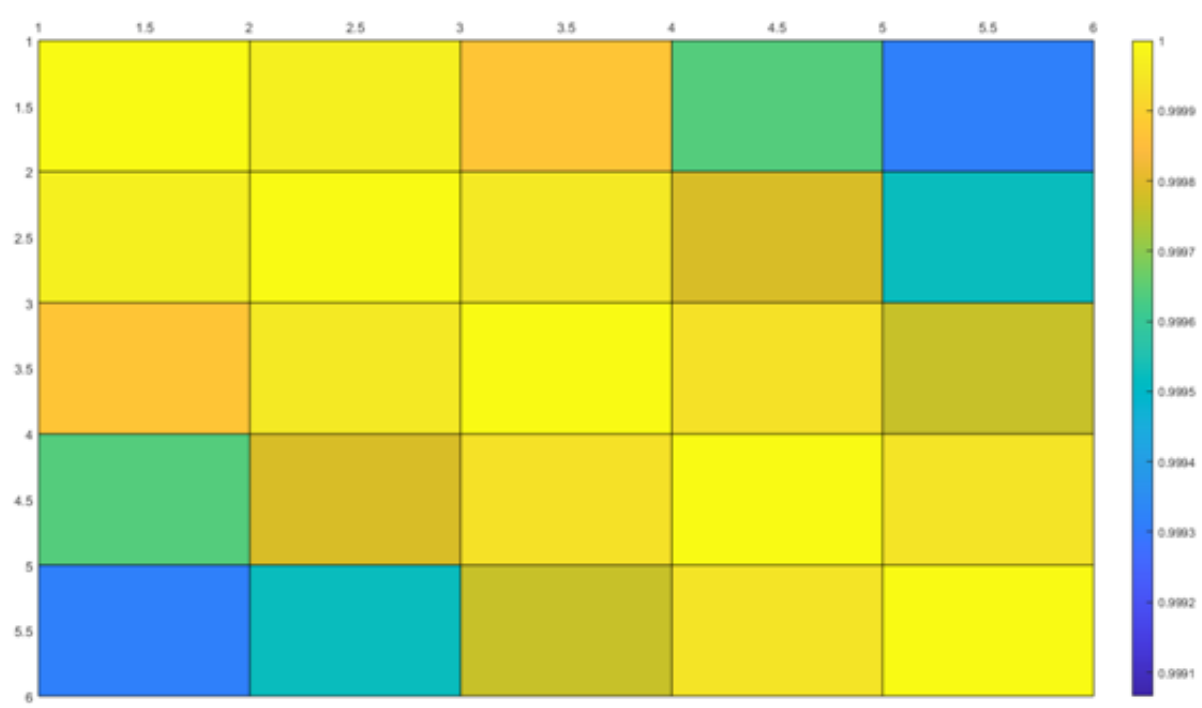

(b)

Figure 2. Three- (a) and two- (b) dimensional plots of the CCs between the six NSs (19-24) in the central NR area, as captured by the corresponding NDs over the entire signal length of the NSs), revealing high CCs (above 0.999 ) over the entire group of NSs/NDs. 


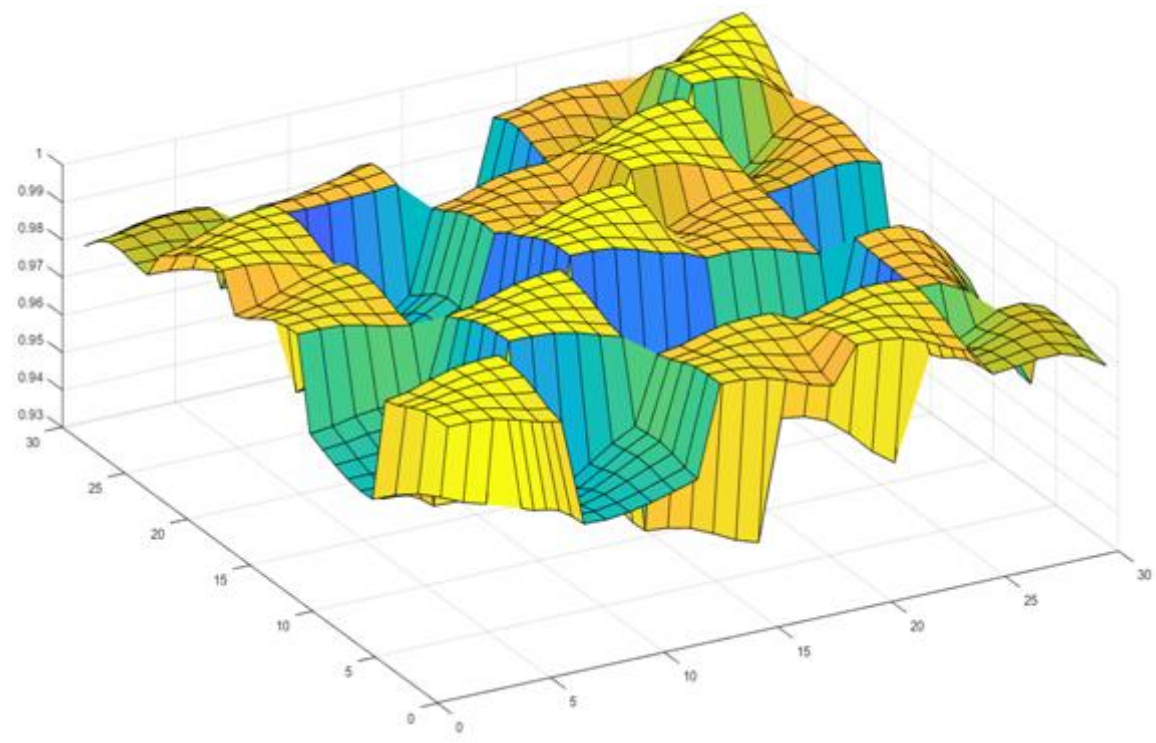

(a)

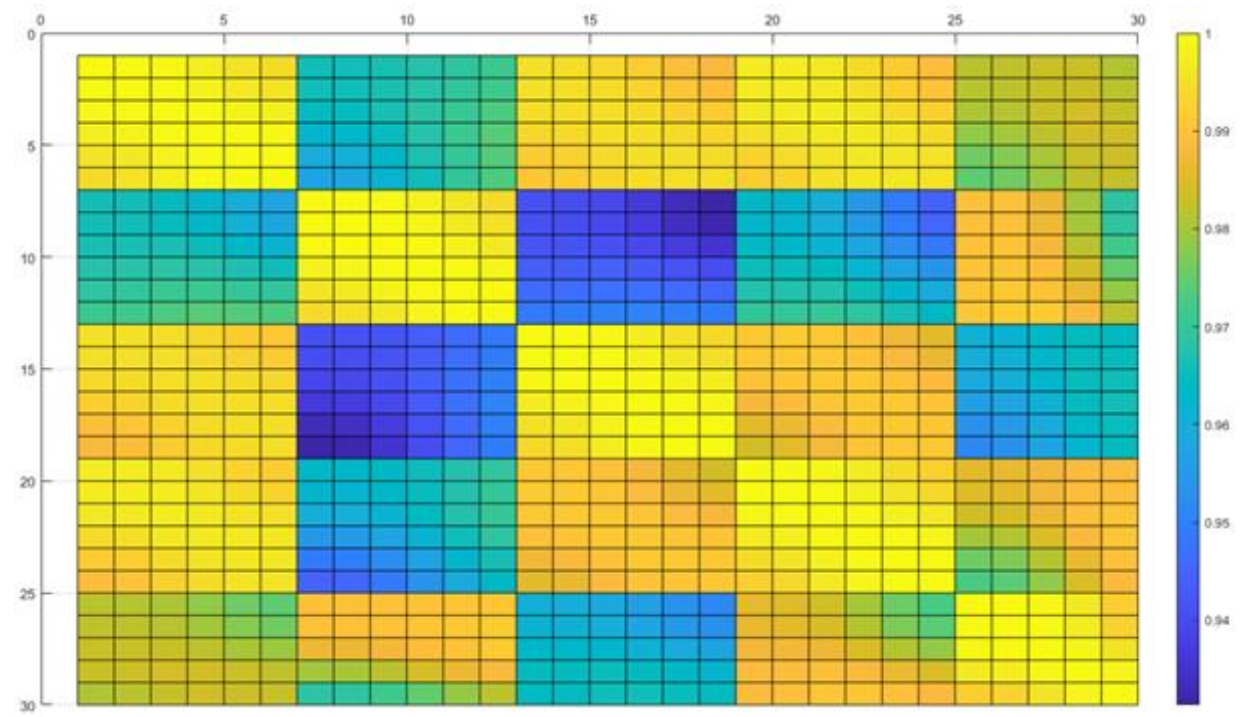

(b)

Figure 3. Three- (a) and two- (b) dimensional plots of the CCs between the thirty NSs (1-18 and 25-36) in the peripheral NR area, as captured from the corresponding NDs over the entire signal length of the NSs, revealing high $\mathrm{CCs}$ (well above 0.9 ) over the entire group of NSs/NDs.

\section{On-Line Selection of the Minimal Set Of 3-Tuples of Neutron DETECTOR/Signals FOR OPTIMAL OLM OF THE ENTIRE SETS OF NEUTRON DETECTORS/NEUTRON NOISE SigNALS}

\subsection{Problem Representation}

As mentioned in the Introduction, a significant improvement of the proposed implementation over the original use of the 3-tuple methodology is the selection of a maximally information-rich pair of in-NSs/in-NDs for the first two (constant) members of each 3-tuple of any given 6x6 sub- 
International Journal of Artificial Intelligence and Applications (IJAIA), Vol.11, No.5/6, November 2020

area, with the third member of each of these 3-tuples being a distinct NS from the remaining four $\mathrm{NSs}$, in turn, thereby reducing the computational (time- and space-) complexity

Table I: Comparative evaluation of the sets of NDs/NSs 1-6, 7-12, 13-18, 19-24, 25-30, 31-36 according to criteria (CRIT.1) through to (CRIT.4) ${ }^{8}$, with pairs $(3,4),(9,10),(15,16),(21,22),(27,28)$ and $(33,34)$ selected as the "constant pairs" of the 3 -tuples and the remaining NDs/NSs constituting the third members of the 3-tuples.

\section{Sets of NDs/NSs 1-6}

\begin{tabular}{|l|c|c|c|c|c|c|}
\hline $\mathrm{ND} / \mathrm{NS}$ & 1 & 2 & $\mathbf{3}$ & $\mathbf{4}$ & 5 & 6 \\
\hline $\min \mathrm{CC} \uparrow$ & 0.9946 & 0.9956 & $\mathbf{0 . 9 9 7 6}$ & $\mathbf{0 . 9 9 7 8}$ & 0.9959 & 0.9946 \\
\hline meanCC $\uparrow$ & 0.9975 & 0.9981 & $\mathbf{0 . 9 9 8 9}$ & $\mathbf{0 . 9 9 8 9}$ & 0.9981 & 0.9974 \\
\hline $\max \mathrm{CC} \uparrow$ & $\mathbf{0 . 9 9 9 9}$ & $\mathbf{0 . 9 9 9 9}$ & 0.9997 & 0.9997 & $\mathbf{0 . 9 9 9 9}$ & $\mathbf{0 . 9 9 9 9}$ \\
\hline std CC $\downarrow$ & 0.0020 & 0.0017 & $\mathbf{0 . 0 0 0 8}$ & $\mathbf{0 . 0 0 0 7}$ & 0.0016 & 0.0020 \\
\hline
\end{tabular}

\section{Sets of NDs/NSs 7-12}

\begin{tabular}{|c|c|c|c|c|c|c|}
\hline ND/NS & 7 & 8 & 9 & 10 & 11 & 12 \\
\hline $\min \mathrm{CC} \uparrow$ & 0.9944 & 0.9952 & 0.9970 & 0.9982 & 0.9962 & 0.9944 \\
\hline meanCC $\uparrow$ & 0.9977 & 0.9981 & 0.9988 & 0.9990 & 0.9982 & 0.9970 \\
\hline $\max C \mathrm{C} \uparrow$ & 0.9999 & 0.9999 & 0.9997 & 0.9996 & 0.9997 & 0.9997 \\
\hline std $\mathrm{CC} \perp$ & 0.0021 & 0.0018 & 0.0010 & 0.0005 & 0.0014 & 0.0020 \\
\hline
\end{tabular}

\section{Sets of NDs/NSs 13-18}

\begin{tabular}{|l|c|c|c|c|c|c|}
\hline $\mathrm{ND} / \mathrm{NS}$ & 13 & 14 & $\mathbf{1 5}$ & $\mathbf{1 6}$ & 17 & 18 \\
\hline $\min \mathrm{CC} \uparrow$ & 0.9939 & 0.9951 & $\mathbf{0 . 9 9 7 3}$ & $\mathbf{0 . 9 9 7 5}$ & 0.9953 & 0.9939 \\
\hline meanCC $\uparrow$ & 0.9972 & 0.9978 & $\mathbf{0 . 9 9 8 8}$ & $\mathbf{0 . 9 9 8 8}$ & 0.9979 & 0.9971 \\
\hline max CC $\uparrow$ & $\mathbf{0 . 9 9 9 9}$ & $\mathbf{0 . 9 9 9 9}$ & 0.9996 & 0.9996 & $\mathbf{0 . 9 9 9 9}$ & $\mathbf{0 . 9 9 9 9}$ \\
\hline std CC $\downarrow$ & 0.0023 & 0.0019 & $\mathbf{0 . 0 0 0 9}$ & $\mathbf{0 . 0 0 0 8}$ & 0.0018 & 0.0023 \\
\hline
\end{tabular}

\section{Sets of NDs/NSs 19-24}

\begin{tabular}{|l|c|c|c|c|c|c|}
\hline $\mathrm{ND} / \mathrm{NS}$ & 19 & 20 & $\mathbf{2 1}$ & $\mathbf{2 2}$ & 23 & 24 \\
\hline $\min \mathrm{CC} \uparrow$ & 0.9991 & 0.9993 & $\mathbf{0 . 9 9 9 6}$ & $\mathbf{0 . 9 9 9 6}$ & 0.9993 & 0.9991 \\
\hline meanCC $\uparrow$ & 0.9996 & 0.9997 & $\mathbf{0 . 9 9 9 8}$ & $\mathbf{0 . 9 9 9 8}$ & 0.9997 & 0.9996 \\
\hline $\max \mathrm{CC} \uparrow$ & $\mathbf{1 . 0 0 0 0}$ & $\mathbf{1 . 0 0 0 0}$ & 0.9999 & 0.9999 & $\mathbf{1 . 0 0 0 0}$ & $\mathbf{1 . 0 0 0 0}$ \\
\hline std CC $\downarrow$ & 0.0003 & 0.0003 & $\mathbf{0 . 0 0 0 1}$ & $\mathbf{0 . 0 0 0 1}$ & 0.0003 & 0.0003 \\
\hline
\end{tabular}

\section{Sets of NDs/NSs 25-30}

\begin{tabular}{|c|c|c|c|c|c|c|}
\hline ND/NS & 25 & 26 & $\mathbf{2 7}$ & $\mathbf{2 8}$ & 29 & 30 \\
\hline $\min$ CC $\uparrow$ & 0.9926 & 0.9940 & $\mathbf{0 . 9 9 6 5}$ & $\mathbf{0 . 9 9 7 2}$ & 0.9946 & 0.9926 \\
\hline mean CC $\uparrow$ & 0.9967 & $\mathbf{0 . 9 9 8 5}$ & $\mathbf{0 . 9 9 8 5}$ & $\mathbf{0 . 9 9 8 6}$ & 0.9975 & 0.9963 \\
\hline $\max$ CC $\uparrow$ & $\mathbf{0 . 9 9 9 9}$ & $\mathbf{0 . 9 9 9 9}$ & 0.9996 & 0.9995 & 0.9998 & 0.9998 \\
\hline std $\mathrm{CC} \square$ & 0.0028 & 0.0023 & $\mathbf{0 . 0 0 1 2}$ & $\mathbf{0 . 0 0 0 9}$ & 0.0021 & 0.0027 \\
\hline
\end{tabular}

\footnotetext{
${ }^{8}$ where the auto-CCs have been excluded for the evaluation of max $\mathrm{CC}$
} 


\section{Sets of NDs/NSs 31-36}

\begin{tabular}{|l|c|c|c|c|c|c|}
\hline ND/NS & 31 & 32 & $\mathbf{3 3}$ & $\mathbf{3 4}$ & 35 & 36 \\
\hline $\min \mathrm{CC} \uparrow$ & 0.9864 & 0.9873 & 0.9911 & $\mathbf{0 . 9 9 5 8}$ & $\mathbf{0 . 9 9 2 2}$ & 0.9864 \\
\hline mean CC $\square$ & 0.9949 & 0.9955 & $\mathbf{0 . 9 9 7 0}$ & $\mathbf{0 . 9 9 7 6}$ & 0.9958 & 0.9919 \\
\hline $\max$ CC $\uparrow$ & $\mathbf{0 . 9 9 9 9}$ & $\mathbf{0 . 9 9 9 9}$ & 0.9996 & 0.9991 & 0.9991 & 0.9991 \\
\hline std CC $\square$ & 0.0051 & 0.0040 & 0.0032 & $\mathbf{0 . 0 0 1 2}$ & $\mathbf{0 . 0 0 2 9}$ & 0.0050 \\
\hline
\end{tabular}

of in-ND/in-NS validation, while rendering the process transparent as well as easily adaptable to different requirements and priorities.

\subsection{Level and Means of Problem Encoding}

Among the extensive choice of candidate problem-representations/solutions, the following reasoning has been followed here: the 3-tuples are ranked in terms of the following four information-content criteria, namely highest values for the minimum (CRIT.1), mean (CRIT.2) and maximum (CRIT.3) pairwise CC values of the 3-tuple, as well as lowest value for the standard deviation (std, CRIT.4) of the pairwise CC values of the 3-tuple. The combination (in the straightforward as well as computationally simple form of the sum/average ${ }^{9}$ ) of the ranks over the four NS information-content criteria promotes the selection of NSs/NDs which - further to demonstrating as high as possible CCs with the NSs of their cluster - exhibit a relatively uniform similarity measure, i.e. comparable distances from the other NSs of the cluster. For the present implementation, CRITs.1-4 have been assigned equal weighing, as there is no evidence either of any criterion having higher priority over another, or of the necessity for a more complicated weighing scheme.

Table I shows the numerical values of each candidate ND/NS of every cluster, in turn, for criteria CRIT.1 through to CRIT.4, further indicating the obtained optimal values for each cluster (highlighted in the Table). For ease of comprehension, the implemented selection methodology is briefly explained for the set of in-NDs/in-NSs 19 to 24 (sub-Table "sets of NDs/NSs 19-24" of Table I), as the differences in values for the used features corresponding to this in-ND/NS set are small relative to those of other in-ND/in-NS sets, whereby the discriminatory power of the proposed approach can be fittingly highlighted. As shown in this sub-Table, the values of the four criteria for this set of in-NDs/in-NSs support the selection of NDs/NSs 22 and 21 as the "first \& second"/best two (constant) members of the 3 -tuple $^{10}$ (highlighted and marked as bold in the Table), with each of the other in-NDs/in-NSs 19, 20, 23 and 24 constituting the "third" members of the four created 3-tuples, in turn.

It should be mentioned here that the "best" (constant) pair of in-NDs/in-NSs of each 3-tuple is continuously (at every time-step) validated along the STW of the most recent CC values/ window of the in-NSs corresponding to the constant pair, as well as against the CC values of the other inNSs of the sub-area (for the same time-window), for confirming anticipated NR functioning, as well as accurate in-ND/in-NS validation and decision-making. This is essential since, even the slightest deviation between a pair of signals - especially if it exceeds the desired NN leveldependent, statistically accepted, limit for the current mode of NR operation - must be directly detected and compared with the remaining in-NDs/in-NSs that are monitored by the constant

\footnotetext{
${ }^{9}$ CRIT(s).1-4 receive equal weighing as there is no evidence, either of any criterion having higher priority over another, or of the necessity for a more complicated weighing scheme

${ }^{10}$ since they demonstrate the highest values for the two criteria min $\mathrm{CC}$ and mean $\mathrm{CC}$, the lowest value for the criterion min $\mathrm{CC}$ and next-best value for the criterion max $\mathrm{CC}$
} 
pair, so that (ii) any member of the constant in-ND/in-NS pair that is found to behave abnormally (due to ND malfunctioning or some other exogenous NR condition) can be substituted by the next-best in-ND/in-NS, with the CC values and thresholds for in-ND/in-NS validation being adjusted accordingly.

It is also important that, owing to the option of selecting the window length according to the current flow-regime and/or mode of operation, the appropriate time-window (or a limited set of alternative time-windows) can be applied to the in-NSs for accommodating (and, thus, explaining) changes in data characteristics, as these develop at any location as well as at any time during NR operation.

\subsection{Implementation - Results}

In all, 12 in-NDs/in-NSs NSs/NDs (two per sub-area) have been found adequate for performing OLM of the entire NR in-core area. The modularity afforded by the proposed construction allows each (sub-)area to be monitored, tested and reconfigured independently of the others, something that is valuable when different sub-areas present different in-NDs/in-NSs NS/ND behaviour or abnormalities, i.e. where and when the different evolving OLM characteristics require distinct methodologies and/or asynchronous time-windows for the optimization of each ND/NS configuration. It is also important that the 3-tuple set-up can be directly reformulated as soon as a sequence of STW-evaluated CCs is detected to deviate from the other CCs involving the same constant in-ND/in-NS pair. The interested reader is referred to [1] for a quantitative presentation of the effects of different in-NS- and/or in-ND-originating "faults" (from both deviating in-NSs or malfunctioning/failing in-NDs, respectively), as well as a numerical comparison of the 3 -tuple configuration against polynomial approximation [8] and signal representation via (semiparametric) splines [9].

Table II: Comparative evaluation of the sets of NDs/NSs 1-6, 7-12, 13-18, 19-24, 25-30, 31-36 according to criteria (CRIT.1) through to (CRIT.4) ${ }^{11}$, with pairs $(3,4),(9,10),(15,16),(21,22),(27,28)$ and $(33,34)$ selected as the "constant pairs" of the respective 3 -tuples and the remaining NDs/NSs constituting the third members of the 3-tuples.

\begin{tabular}{|c|c|c|}
\hline GRNN inputs & \multicolumn{2}{|c|}{$\begin{array}{c}\text { in-NS3 \& in-NS4 evaluation/prediction of in-NS2 vs } \\
\text { collected in-NS2 }\end{array}$} \\
\hline ND/NS correct operation & erroneous in-NS2 & malfunctioning in-ND2 \\
\hline normal & $1.9768 \mathrm{e}-04$ & $1.9768 \mathrm{e}-04$ \\
& & 0.3084 (in-ND3 $\times$ ) \\
& 0.0042 & 0.0338 (in-ND4 $\times$ ) \\
\hline TS (small trend) & 0.0053 & 0.3473 (in-ND3 $\times$ ) \\
& & 0.0389 (in-ND4 $\times$ ) \\
\hline TL (large trend) & $3.4274 \mathrm{e}-11$ & $4.026 \mathrm{e}-06$ (in-ND3 $\times$ ) \\
& & $8.753 \mathrm{e}-07$ (in-ND4 $\times$ ) \\
\hline OS (small oscillation) & $0.3395 \mathrm{e}-08$ & $0.3084-07$ (in-ND3 $\times$ ) \\
& & $0.0338-08$ (in-ND4 $\times$ ) \\
\hline OL (large oscillation) & 0.0051 & 0.0985 (in-ND3 $\times$ ) \\
& & 0.1638 (in-ND4 $\times$ ) \\
\hline OT (small trend and & &
\end{tabular}

\footnotetext{
${ }^{11}$ where the auto-CCs have been excluded for the evaluation of $\max \mathrm{CC}$
} 
Following the same rationale, the results of the operation of the in-NS 1, in-NS 3, in-NS 2$\} 3$ tuple, which appear in [1, p.530], can be compared to the results of the present implementation ${ }^{12}$ (Table II) which uses the constant pair \{ in-NS 3, in-NS 4$\}$ of the 3-tuple for testing the remaining in-NSs, demonstrating the improvement realized by the most general case of using an "optimal" constant pair of in-NDs/in-NSs for determining normal/anticipated NS collection of the remaining in-ND/in-NS, respectively. The differences in the responses of the 3-tuple between deviating in-NSs and failing in-NDs are displayed, while a comparison of the general regression artificial neural network (GRNN [8] implementation with alternative methodologies (polynomial approximation [9] and semi-parametric splines [10]) for combining 3-tuples for a constant pair of in-ND/in-NS $1 \& 3$ for 2 appears in [1], which - combined - support the accuracy as well as efficiency afforded by the in-NS/ in-ND-"pair per sub-area" implementation, as described above.

It is important that the proposed methodology is directly reconfigurable: the CCs between the six in-NSs of each cluster are constantly monitored, whereby the currently defined as constant pair of the 3-tuple changes automatically once the pairwise CCs of another pair exceed those of the currently constant pair; a time-window is used in this case, during which both CCs are recorded for avoiding oscillations between constant pairs.

Future research will focus upon the application of wavelet analysis [11] to the in-NSs, which is expected to complement the presented approach for concurrently capturing malfunctioning NDs, as well as erroneous NSs, via the simultaneous application of frequency analysis and localization in space of sources of anomaly (be it deviating NSs or malfunctioning NDs), as afforded by this combination of methods.

\section{Conclusions}

The presented research has introduced a purely data-driven, directly reconfigurable, divide-andconquer, OLM methodology for automatically selecting the minimum number of NDs - and corresponding NSs - which are currently necessary, as well as sufficient, for inspecting the entire nuclear reactor (NR) in-core area. The proposed implementation has been based on the 3-tuple configuration, according to which a set of three sufficiently pairwise-correlated NSs is capable of on-line (I) verifying each NS of the 3-tuple and (II) endorsing correct functioning of each corresponding ND via straightforward pairwise comparisons of the fixed-length synchronized STWs of the 3-tuple of NSs. A key-modification/advancement of the original 3-tuple configuration has been implemented - made possible by the sufficiently high CCs obtained between the in-NDs/in-NSs of the same cluster (sub-area) -, which allows the cooperation of a single pair of sufficiently CC-ed in-NDs/in-NSs which are also adequately CC-ed with the remaining four in-NDs/in-NSs of the cluster for on-line monitoring the entire in-core area. Owing to the non-homogeneous nature of the in-core area, a divide-and-conquer approach has been implemented for determining the minimum number of NDs and the corresponding NSs which are needed for detecting malfunctioning NDs as well as deviating NSs (either from the norm or from the neighbouring NSs). Tests on the in-core dataset of the PWR model confirm the potential of the proposed methodology in terms of ND/NS selection accuracy, efficiency and robustness, while further establishing its adaptability to changing NR operating conditions and demonstrating its "directly reconfigurable" property in case of NS distortion or failure, and/or ND malfunctioning.

\footnotetext{
${ }^{12}$ Using the alternative ("best" under the currently implemented criteria) in-NS 3 -tuple $\{3,4,2\}$, i.e. employing ND/NS3 and ND/NS4 for monitoring ND/NS2.
} 
International Journal of Artificial Intelligence and Applications (IJAIA), Vol.11, No.5/6, November 2020

\section{NOMENCLATURE}

\begin{tabular}{|c|c|}
\hline CC & cross-correlation \\
\hline HT ANN & harmony theory artificial neural network \\
\hline in-/ex- & in-core/ex-core \\
\hline ND & neutron detector \\
\hline NN & neutron noise \\
\hline NR & nuclear reactor \\
\hline NS & neutron noise signal \\
\hline OLM & on-line monitoring \\
\hline PWR & pressurized water nuclear reactor \\
\hline STW & sliding time-window \\
\hline
\end{tabular}

\section{ACKNOWLEDGEMENTS}

The research leading to these results (i) has received funding from the H2020 CORTEX Euratom Research and Training programme 2014-2018 under grant agreement No 754316, (ii) is supported by the Research Office of the University of Piraeus. The author further wishes to thank the Editors-in-Chief and the Reviewers of the Journal for their pertinent comments, which have helped to significantly improve this submission.

\section{REFERENCES}

[1] Tambouratzis Tatiana, Chionis Dionysios, Dokhane Abdelhamid, (2018). General Regression Neural Networks for the Concurrent, Timely and Reliable Identification of Detector Malfunctions and/or Nuclear Reactor Deviations from Steady-State Operation, in Proceedings of the "2018 IEEE Symposium Series on Computational Intelligence (SSCI)", Bengaluru, India, November 18th-21st, 2018, pp. 524-531.

[2] Rabiner L.R., Gold B. (1979). Theory and Application of Digital Signal Processing, pp. 401, Prentice-Hall Inc., Englewood Cliffs, NJ, U.S.A. (1975).

[3] Tambouratzis T., Pantera L., Stulik P. (2020). A Study on the Minimum Requirements for the OnLine, Efficient and Robust Validation of Neutron Detector Operation and Monitoring of Neutron Noise Signals Using Harmony Theory Networks, in Proceedings of the "2nd International Conference on Machine Learning \& Applications (CMLA 2020)", Copenhagen, Denmark, September 26th-27th, 2020.

[4] Glasstone S., Sesonkse A. (1994). Nuclear Reactor Engineering, Chapman and Hall.

[5] Demaziere Ch., Dokhane A. (2019). Description of Scenarios for the Simulated Data, Technical Report CORTEX Research and Innovation Action (RIA).

[6] http://cortex-h2020.eu (Horizon 2020 Programme, Research and Innovation Action (RIA), grant agreement No 75416).

[7] Smolensky P. (1986). Information Processing in Dynamical Systems: Foundations of Harmony Theory, in Parallel Distributed Processing, vol. 1. Foundations (Rumelhart D.E., McClelland J.L. eds), pp. 194-281.

[8] Specht, D.F. (1991). A General Regression Neural Network, IEEE Transactions on Neural Networks, vol. 2., pp. 568-576.

[9] Phillips G.M., Taylor P.J. (1996). Theory and Applications of Numerical Analysis (2nd Edition), Academic Press, Elsevier. 
International Journal of Artificial Intelligence and Applications (IJAIA), Vol.11, No.5/6, November 2020

[10] Ahlberg J.H., Nilson E.N., Walsh J.L. (1967). The Theory of Splines and Their Applications, Academic Press.

[11] Mallat S. (1999). A Wavelet Tour of Signal Processing, Academic Press, Elsevier.

\section{AUTHOR}

\section{Tatiana Tambouratzis}

\section{Short Biography}

\section{Education - Positions}

- BSc in Mathematics (University of Athens, Greece), MSc in Intelligent Systems (Brunel University, U.K.) and Ph.D. in Artificial Neural Networks and Artificial Vision (Brunel University, U.K.)

- $\quad$ Researcher Grade IV, III, II (Institute of Informatics and Institute of Nuclear Technology - Radiation Protection, NCSR Demokritos, Attiki, Greece)

- Assistant and Associate Professor (Department of Industrial Management \& Technology, University of Piraeus, Piraeus, Greece)

- Visiting Researcher (Department of Nuclear Engineering, Chalmers University of Technology, Göteborg, Sweden)

\section{Scholarships}

- The Greek Scholarship Foundation (Greece)

- The Science and Engineering Research Council (SERC) (U.K.)

- The National Research Institute (Greece)

- $\quad$ The Royal Society (U.K.)

- The Fulbright Teaching Mobility Scholarship Program (U.S.A.)

\section{Memberships/Boards}

- Fellow of the Institute of Mathematics and its Applications (IMA), membership no. 23668

- Member of the Institute of Electrical and Electronics Engineers (IEEE), membership no. 93994512

- Member of the Editorial Advisory Board of Progress in Nuclear Energy (area: Fission Technology) 